\title{
Structural Properties and vulnerability of Iranian 400kv Power Transmission Grid: a Complex Systems Approach
}

\section{M.A.S. Monfared ${ }^{1 *}$ and Zohre Alipour ${ }^{2}$}

${ }^{1}$ M.A.S. Monfared, Associate Professor, College of Engineering, Alzahra University, Tehran, Iran ${ }^{2}$ Zohreh Alipour, Ph.D student, Faculty of Industrial Engineering, Tehran University, Tehran, Iran

\begin{abstract}
We investigate, for the first time, the structural properties of the very high voltage $(400 \mathrm{kv})$ power transmission network of Iran. Researches in complex systems theory have suggested that topology of the most real networks display power law shaped degree distribution, as a signature of complex networks. However, observations shed doubt on this as the degree distribution of power grids studied in different countries display exponential degree distribution rather than power law distribution (fat tailed shapes). We perform our analysis on Iran's power grid to provide more evidence for this interesting and important result. In addition, we provide some analysis for assessing the vulnerability and reliability of the power grid.
\end{abstract}

Keywords: Complex systems; Topology analysis; Power grid; Reliability; Vulnerability.

\section{Introduction}

Many natural systems such as the human brains and food chains, engineering systems such as the Internet, and the power grids and social systems such as network of actors and the network of scientists are making only a few examples of networks composed by a large number of highly nonlinear elements, which are called complex networks. Researches on complex networks are undertaken in many disciplines including physics, biology, economics, engineering and social science. It is not surprising that the complex networks are such a broad area of research that it is even difficult for scientists to present a single concise definition [1].

Unlike in many scientific situations, the elements in a complex network are not often identical, may have complex structures, and perhaps not have strictly defined roles. Moreover, there are frequently stochastic components to the interaction and external noise acting on the units. Complex networks are typically out-of-equilibrium [2,3] due to poorly characterized external perturbations imposed on elements and highly nonlinear correlations among elements[4-6].

The idea that dynamical behavior of complex networks could be strongly influenced by the structure or the topology of network was originally studied by Watts and Strogatz [7] ,on the development of small-world networks. Then it $s$ followed by Barabasi and Albert, on the development of scale-free networks [8]. The importance of network topology in real complex networks such as power grids was further recognized by the presence of hierarchical sub structures or communities which could not been anticipated within the classical theory of regular and random graphs $[9,10]$.

Power grids praised as the most complex engineering innovation entails properties which add to its importance: it has grown steadily over the last decades and will continue its course in future [2]; often display correlations between events suggesting that the system memory and its dynamics are important; exhibit infrequent large cascading failures [3]. Such properties suggests that conventional RAMS, i.e. reliability, availability, maintainability, and safety methodologies do not apply to these systems $[11,12]$, and new methodologies needs to be developed.

The first attempt to capture the topological properties of such systems is to model them as graphs whose nodes represent the dynamical units, and whose links stand for the interactions between them [13]. Historically, the study of graphs has been mainly the domain of a branch of discrete mathematics known as graph. In addition to the developments in mathematical graph theory, the study of networks has seen important achievements in some specialized contexts, as for instance in the social science. In particular the last decade has witnessed the rebirth of interest and research in the study of complex networks in physics' community, computer science, and engineering among others [14].

The number of books and articles that investigate complex networks is growing, e.g. $[9,14,15]$. Research works on power grids have demonstrated that the topology of an electric power grid tells us important information on the dynamical behavior as well the vulnerability of the network. See for examples, Watts and Strogatz [16], Barabasi and Albert [17], Crucitti et al. [18], Albert et al. [19], Chassin et al. [4], Rosato et al. [5], Casals et al. [2,6]. However, results obtained so far from the analysis of topological properties of power grids shed doubt on what is considered as a signature of complex systems, i.e. complex systems exhibits power law degree distribution.

Researches in complex systems theory have suggested that topology of the most real networks display power law shaped degree distribution, in addition to small path lengths, high clustering coefficients, correlated node degrees, and the presence of hierarchical sub structures or communities [13]. In this article, we investigate for the first time the structural properties of 400kvpower transmission network in Iran to provide new evidence for this interesting and important debate. In so doing, we also provide analysis for assessing the vulnerability of the

*Corresponding author: Monfared MAS, associate professor, School of Engineering, Alzahra University, Vanak Street, Tehran, Iran, E mail: mas_monfared@alzahra.ac.ir

Received January 25, 2013; Accepted May 17, 2013; Published May 22, 2013

Citation: M.A.S. Monfared, Alipour Z (2013) Structural Properties and vulnerability of Iranian 400kv Power Transmission Grid: a Complex Systems Approach. Ind Eng Manage 2: 112. doi:10.4172/2169-0316.1000112

Copyright: () 2013 M.A.S. Monfared, et al. This is an open-access article distributed under the terms of the Creative Commons Attribution License, which permits unrestricted use, distribution, and reproduction in any medium, provided the original author and source are credited. 
grid and propose some hints for improving the safety and reliability of the power grid in Iran.

This paper is organized as follows. Section 2 is about computing of structural measures of complex networks. Section 3 analyzes the structure of Iran's power transmission network, and compares the results with some European power grids. Section 4 provides some clues for assessing the vulnerability of the power grid. Section 5 gives a conclusion.

\section{The Structure of Complex Networks}

We shall first introduce definitions and notations, and discuss the basic quantities used to describe the topology of a network. A complex network can be represented as a graph. A undirected (directed) graph $\mathrm{G}=(\mathrm{N}, \mathrm{L})$ consists of two sets $\mathrm{N}$ and $\mathrm{L}$ is a set of unordered (ordered) pairs of elements of $\mathrm{N}$. The elements of $\mathrm{N} \equiv\left\{\mathrm{n}_{1}, \mathrm{n}_{2}, \ldots, \mathrm{n}_{\mathrm{N}}\right\}$ are called nodes of the graph $G$, while the interaction between two nodes $L \equiv\left\{l_{1}\right.$, $\left.1_{2}, \ldots, 1_{\mathrm{K}}\right\}$ are called links [20].

A graph $\mathrm{G}=(\mathrm{N}, \mathrm{L})$ can be described by the adjacency matrix $\mathrm{A}$, a $\mathrm{N}$ $\times \mathrm{N}$ square matrix, $\mathrm{a}_{\mathrm{ij}}(\mathrm{i}, \mathrm{j}=1, \ldots, \mathrm{N})$ is equal to 1 when the link $\mathrm{l}_{\mathrm{ij}}$ exists, and zero otherwise [13].

There are some concepts and measures to characterize the topology and analyze real networks, such as the degree of a node (k), The average degree of all nodes $(<\mathbf{k}>)$, The maximum degree as the degree of the node that has the maximum number of connections (hub) $\left(\mathrm{k}_{\max }\right)$, The degree distribution $\mathrm{P}(\mathrm{k})$, The shortest path between any two nodes, The graph diameter as the maximum value of shortest path between all nodes (d), The clustering coefficient, The sparseness as the number of edges of a graph in compare with a graph by the maximal number of edges $\left(\mathrm{S}_{\mathrm{p}}\right)$, described in Table 1 .

\section{The Power Transmission Grid}

Iran power transmission network, like its counterparts in other countries, is growing steadily on an average basis at the rate of $10 \%$ annually [21]. According to the data published by [21] 34\% of power outage in Iran in year 2009 is associated with problems in transmission lines, where $56 \%$ is due to problems in power generation, and $11 \%$ is due to unprecedented events in distribution network. The dynamics behind these ratios are complex due to the factors affecting both time and space.

One major cause of dynamics and complexity in transmission lines stems from age distribution. At Iran power transmission $63 \%$ of lines are young with less than 15 years of age, while at the same time, there are $10 \%$ of lines which are more than 30 years old [13]. This produces both availability and maintainability issues complicating the dynamics of power grids. Age distributions are also different at various levels of power grid. This adds to the dynamics and complexity of power grid.

From structural standpoint, power grid in every country has evolved in years to connect generation sites to consumption centers within its own borders, i.e. each power grid is engineered in a sequential approach to cope with specific economic situations, population distributions, geographical morphologies and industrial requirements among other factors. The structural properties of power grids has attracted attentions from the complex systems researchers in recent years, e.g. $[4,16,17,19]$ in north America, $[2,5,6,18]$ in Europe, $[22,23]$ in China, and [24] in Brazil. In this paper, we provide analysis for structural properties of power grid in Iran, as the second attempt in Asian countries and the first attempt in Iran.

\section{Data for structural analysis}

We have extracted data for our analysis of the topological properties of Iran's power grid using an official document updated recently (in year 2011) containing the detailed description of the whole power grid comprising lines from $400 \mathrm{kv}$ down to $33 \mathrm{kv}$ [25]. A part of this drawing which entails the subnet of north eastern province of Khorasan is shown in Figure 1 (only names on bus bars has been changed from Farsi transcript into English transcript).

According to the data extracted from this drawing and from [21], the power transmission network in Iran has 375 bus bars or nodes (including three types of power generating stations, transmitting substations and distribution substations) and 546 edges (or transmission lines summing up to more than $108960 \mathrm{~km}$ ). The transmission lines in Iran's grid operate at different voltage levels, i.e. $400 \mathrm{kv}, 230 \mathrm{kv}, 132 \mathrm{kv}, 63 \mathrm{kv}$, and $33 \mathrm{kv}$. Details are presented in Table 2 for two subnets of $400 \mathrm{kv}, 230 \mathrm{kv}$.

Now, we will investigate the structural properties of the most important component of the transmission networks, i.e. the high voltage network of $400 \mathrm{kv}$.

\section{Data analysis}

Data in our single line drawing includes nodes and links which have been transformed into a graph using Pajek, a program for large networks analysis [26]. The graph for the high voltage $400 \mathrm{kv}$ power transmission is shown in Figure 2, which is an undirected graph consisting of 105 nodes $(\mathrm{N})$ and 142 edges(L). The results for the degree frequency distribution, which are used here to estimate the mean degree of $\langle\mathrm{k}\rangle$, and the maximum degree of $\mathrm{k}_{\max }$ are computed by Matlab.These are shown in Table 3.

We then performed further analysis on degree frequency distribution to estimate a best fit probability distribution for node degrees of $400 \mathrm{kv}$ power grid.

\section{Degree distribution}

The degree frequency histogram for Iran $400 \mathrm{kv}$ power network is shown at Figure 3.

With graph modeling of high voltage power grid (400 kv), we can analyze Degree distribution of nodes (buses). Figure 4 shows degree of nodes by different color.

Our examination shows that degree distribution can be estimated by an exponential function such as

$$
\begin{aligned}
& \mathrm{P}(\mathrm{k} \geq \mathrm{K})=\sum \mathrm{p}(\mathrm{k}) \\
& \mathrm{P}(\mathrm{k} \geq \mathrm{K})=2.5446 \mathrm{e}^{-\mathrm{k} / 1.587}
\end{aligned}
$$

\begin{tabular}{|l|l|l|}
\hline measures & description & formula \\
\hline $\begin{array}{l}\text { degree } \\
\text { distribution }\end{array}$ & $\begin{array}{l}\text { It is defined the probability of a } \\
\text { node has degree } k .\end{array}$ & $P(k \geq K)=\sum_{k^{\prime}=k}^{\infty} p\left(k^{\prime}\right)$ \\
\hline $\begin{array}{l}\text { mean } \\
\text { shortest } \\
\text { path length }\end{array}$ & $\begin{array}{l}d_{i j} \text { is the length of the geodesic from } \\
\text { node } i \text { to node j in a graph with N } \\
\text { nodes. }\end{array}$ & $<l>=\frac{1}{N(N-1)} \sum_{i, j \in N, i \neq j} d_{i j}$ \\
\hline $\begin{array}{l}\text { clustering } \\
\text { coefficient }\end{array}$ & $\begin{array}{l}\text { The local clustering coefficient is } \\
\text { defined as the ratio between } e_{i} \\
\text { and } k_{i}\left(k_{i}-1\right) / 2, \text { i.e. the maximum } \\
\text { possible number of edges in } G_{i} .\end{array}$ & $c_{i}=\frac{2 e_{i}}{k_{i}\left(k_{i}-1\right)}=\frac{\sum_{j n} a_{j} a_{j m} a_{i n}}{k_{i}\left(k_{i}-1\right)}$ \\
\hline
\end{tabular}

Table 1: Concepts and Measures To Characterize The Topology Of Networks. 


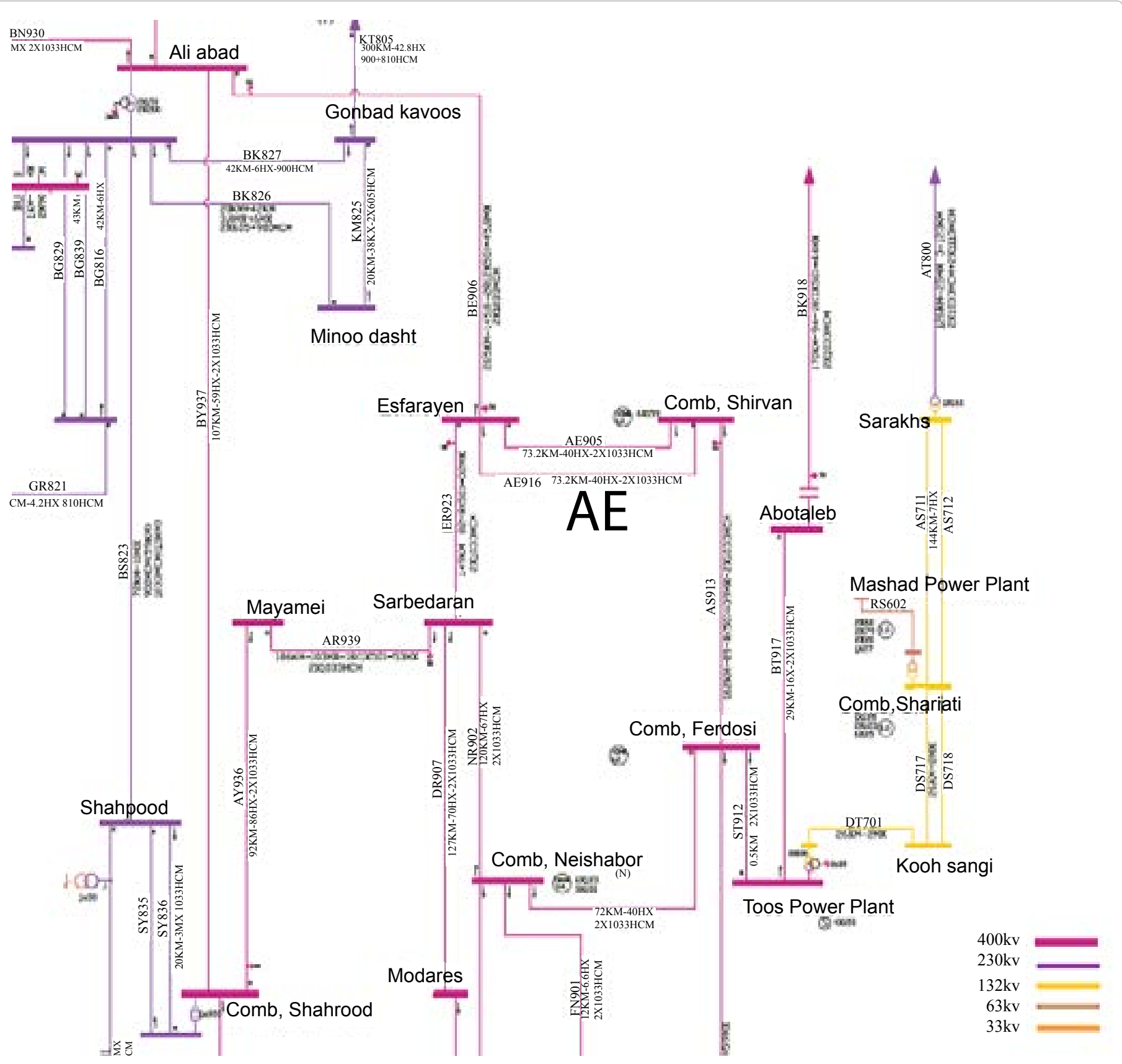

Figure 1: Single line drawingof Iran Power Transmission Network (Khorasan province).

\begin{tabular}{|c|c|c|c|}
\hline voltage level & \# of buses & \# of links & 142 \\
\hline $\mathbf{4 0 0 k V}$ & 105 & 410 \\
\hline $\mathbf{2 3 0} \mathbf{~ k V}$ & 270 & 17,438 \\
\hline
\end{tabular}

Table 2: Specification Of Power Transmission Grid In Iran.

with coefficient of determination of $\mathrm{R}^{2}=0.9626$, as shown in Figure 5. This result agrees with the results reported by Rosato et al. [5]. They have studied topological properties of high voltage electrical power transmission network in three EU countries (i.e. Italian $380 \mathrm{kV}$, the French $400 \mathrm{kV}$, and the Spanish $400 \mathrm{kV}$ networks) and concluded that the degree distributions of different European power grids analyzed are exponential.
The implication is that power grids do not exhibit the degree distribution of scale free networks. This result also agrees with Boccaletti et al. [13] discussing that the scale free networks are good models for all cases in which growth or aging processes do not play a dominant role in determining the structural properties of the network. This is while most power grids are evolving dynamical systems with both growth and aging characteristics. Also Casals et al. [2] have analyzed UCTE 


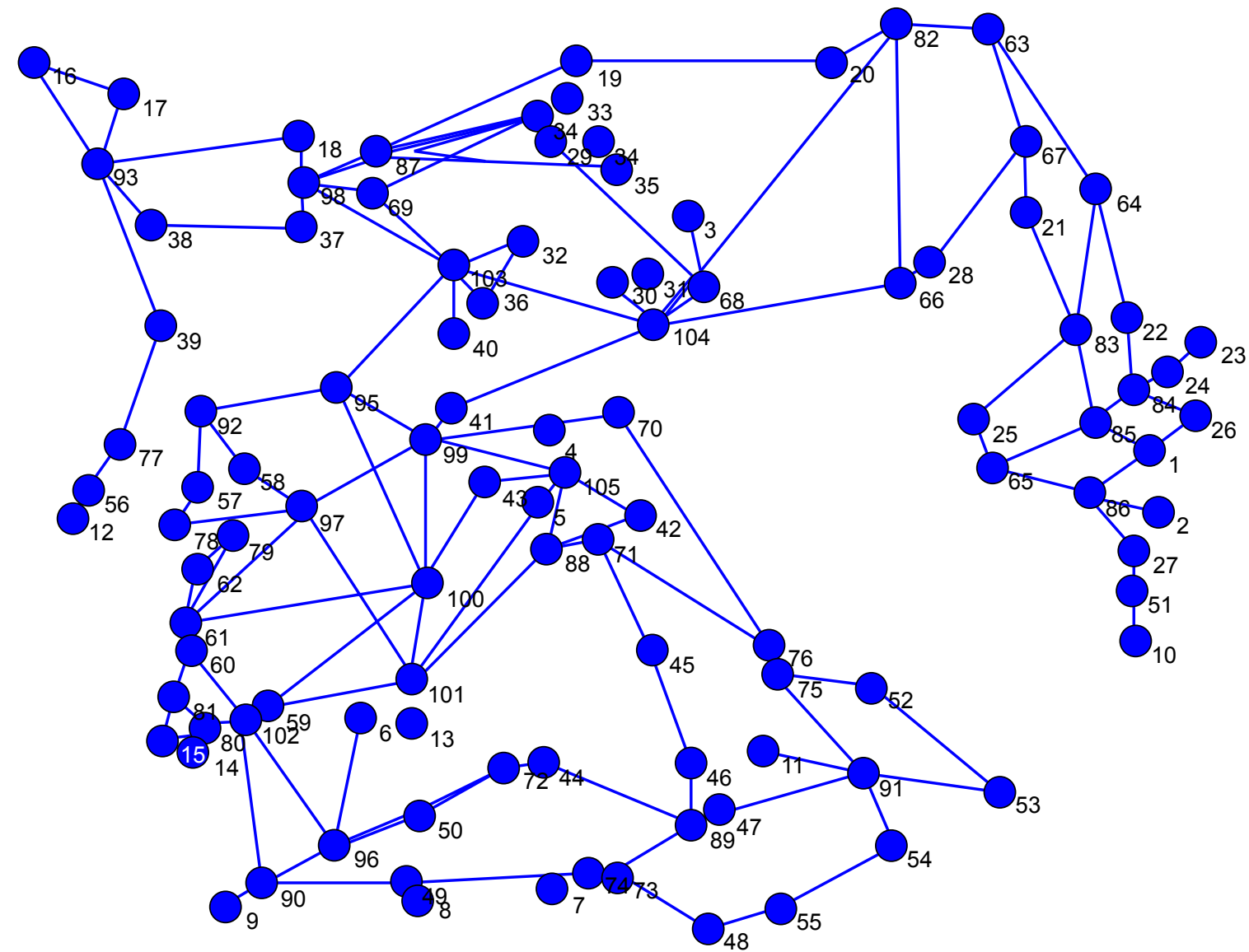

Figure 2: The graph representation of 400 kvhigh voltage power grid.

Transmission network $400 \mathrm{kv}$
$\mathbf{N}$ \begin{tabular}{l|l} 
L & <k>
\end{tabular} 142

2.7048

$<1>$

C 0.1097 $\mathbf{k}_{\max }$

Table 3: Some Topological Specifications Of 400kv Power Grid (N: Number Of Nodes, L: Number Of Edges, <K>: Mean Degree, <L>: Mean Shortest Path, C: Clustering Coefficient, Kmax: Maximum Degree, And D: Graph Diameter).

\section{Degree Histogram}

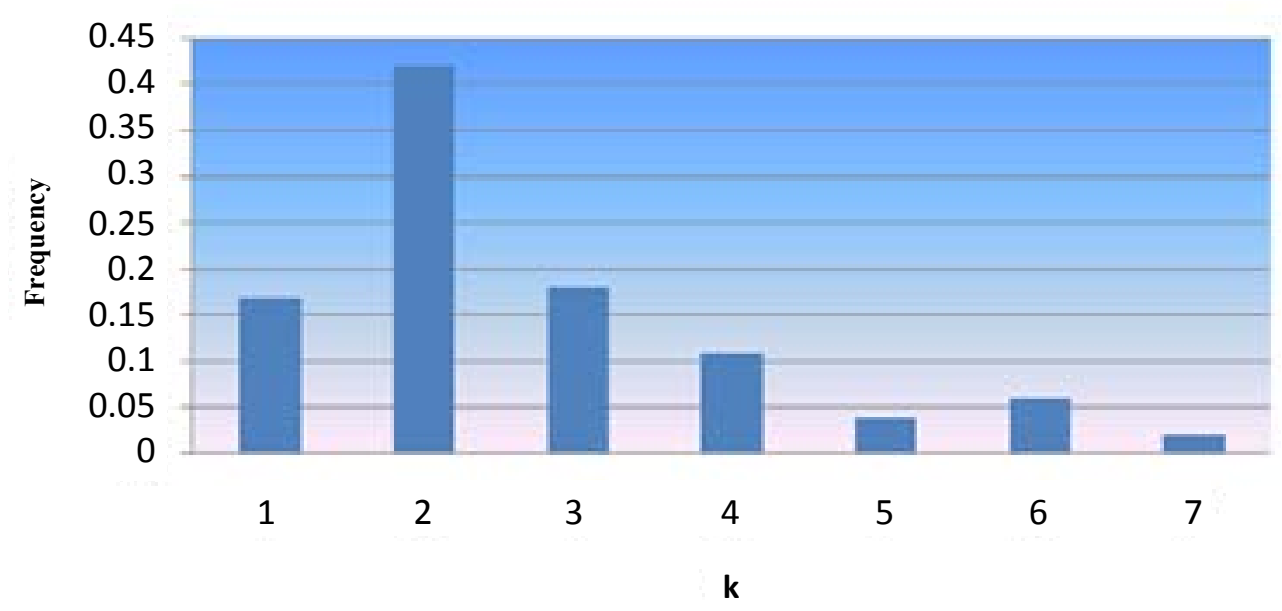

Figure 3: The degree frequency histogram $(400 \mathrm{kv})$ 


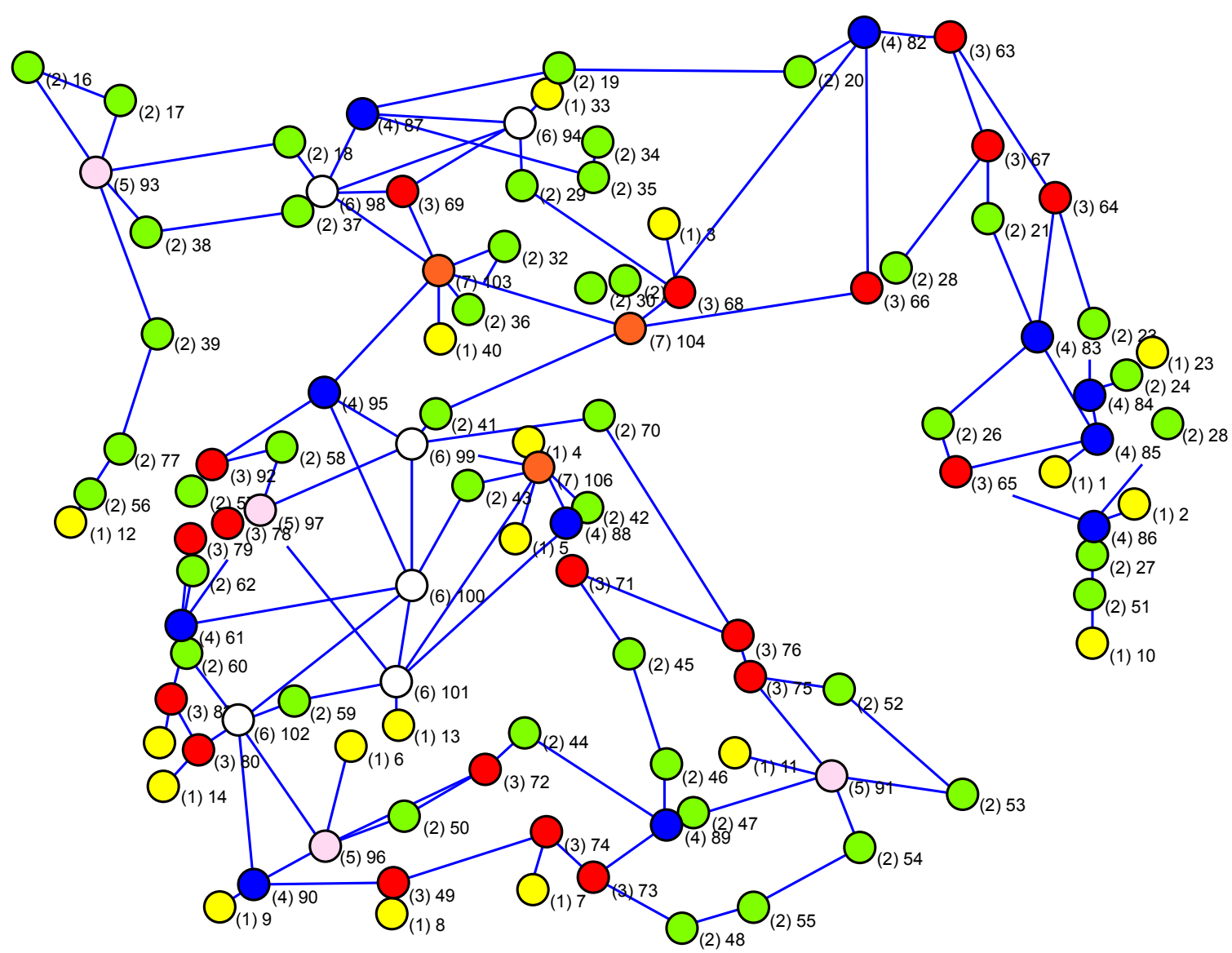

Figure 4: Degree distribution of nodes with different colors (yellow (1), green (2), red(3), blue (4), pink(5), white (6), orange(7)).

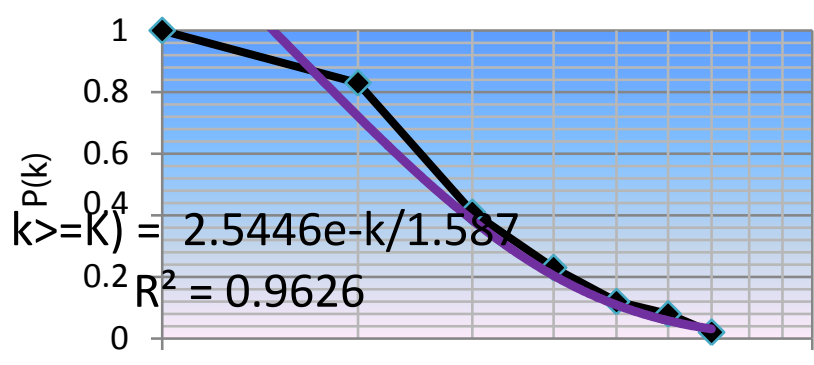

1

10

k

Figure 5: Estimating cumulative degree distribution for 400kv power grid on log-log plot.

power grid, involving thirty three different networks. Every power grid studied has exponential degree distribution, which means that they are not like the highly skewed scale free distribution typically found in other complex networks. In scale free networks, the degree distribution follows a power law.

$$
p(k \geq K)=\sum_{k=K}^{\infty} k^{-\gamma} \sim k^{-(\gamma-1)}
$$

Instead, planar networks in general, and Iran power grid in particular (as well as European power grids), display less skewed exponential degree distribution.

We now collect the results of experiments undertaken in EU countries [5] and compared them with results we have reported in Table 4 to see how Iran's $400 \mathrm{kv}$ power grid behaves.

Data reported in Table 4 displays that Iran high voltage grid is sparse network, with an average degree of 2.7048 , a quite low value with respect to the maximum degree of 7 . Also, this feature is exhibited by the fact that $\mathrm{L}<<\mathrm{N}^{2}$ i.e. $142<<(105)^{2}=11025$. We also note that our 
power grid is heterogeneous. A network is homogeneous (e.g. regular or random network), where nodes degrees are similar, and a network is heterogeneous (e.g. small world network or scale free network) where few nodes (i.e. the hubs) linked to many other nodes, but a large number of nodes are poorly connected.

The relatively moderate value of clustering coefficient of 0.1097 which is extremely larger than random networks, and high value of path length of 6.8817 which is larger than that pertaining to random networks displays the fact that our power grid is classified as small world networks. Hence, the power grids examined by Rosato et al. [5] display topological properties which are typical of small world networks, i.e. they were neither concretely regular networks, nor random networks. Still, they were not scale free networks either, as they do not display power law degree distribution. Still, a recent work by Wang et al. [23] used simulation results of the IEEE - 118 bus system and the central China power grid to show that the cumulative distributions of node electrical betweenness follows a power-law distribution [22]. Furthermore, since the fact that Iran's grid is sparse implies that we can investigate theoretical treatment of the robustness of Iran's power grid using percolation and mean field theories as considered in Section IV.

Figure 6 is a radar diagram exposing the similarities and differences of these four networks. Here, we see that Iran's high voltage power grid shows similar properties with European countries with exceptions two of $\mathrm{C}$ and $<\mathrm{l}>$ which are significantly different. Iran's grid has smaller clustering coefficient, i.e. $\mathrm{C}_{\text {Iran }}=0.1097,<\mathrm{C}_{\text {Italy }}=0.156,<\mathrm{C}_{\text {France }}=0.279$, $<\mathrm{C}_{\text {spain }}=0.316$. On the other hand, Iran's grid shows different mean path lengths as is greater than Italy's case, i.e. $<\mathrm{l}>\operatorname{Iran}=6.8817<<\mathrm{l}>\mathrm{Italy}=8.47$, but at the same time is less than France's case and Spain's case, i.e. $<\mathrm{l}>$ Iran $=6.78><\mathrm{l}>$ France $=6.61><\mathrm{l}>$ Spain $=4.92$.

\section{Vulnerability Analysis}

One way to perform deeper analysis in revealing the complexity of a network is to find sub nets and motifs within a network. Finding the subnets within a network is a powerful tool for understanding the

\begin{tabular}{|c|c|c|c|c|c|c|c|}
\hline Network & $\mathrm{N}$ & $\mathrm{L}$ & $<\mathrm{k}>$ & $<\mathrm{l}$ & $\mathrm{C}$ & $\mathrm{k}_{\max }$ & $\mathrm{d}$ \\
\hline Iran(400kv) & 105 & 142 & 2.7048 & 6.8817 & 0.1097 & 7 & 19 \\
\hline Italian (380kv) & 127 & 171 & 2.69 & 8.47 & 0.156 & 7 & 25 \\
\hline French (400kv) & 146 & 223 & 3.05 & 6.61 & 0.279 & 8 & 15 \\
\hline Spanish(400kv) & 98 & 175 & 3.57 & 4.92 & 0.316 & 9 & 11 \\
\hline
\end{tabular}

Table 4: Iran's Power Transmission Network Topological Specifications.
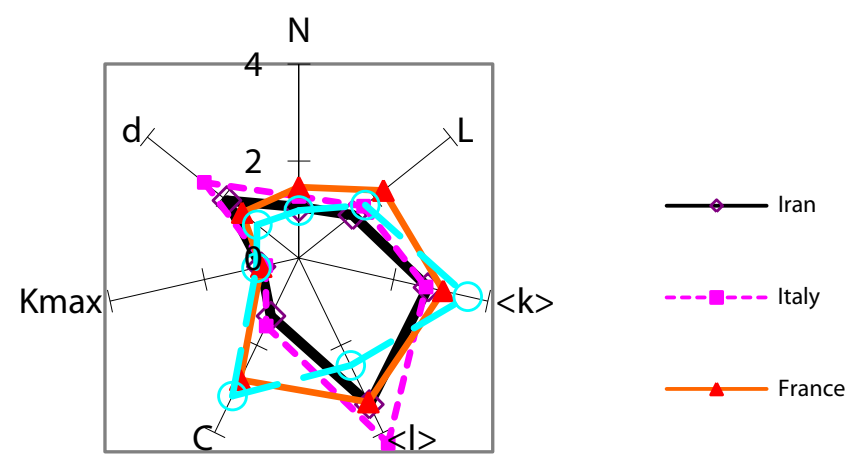

$-\ominus-$ Spain

Figure 6: Radar diagram exposing variations of network measures in four countries(scales have been normalized to enable better expositions): Iran(diamond), Italy(square), France(triangle), and Spain(circle).

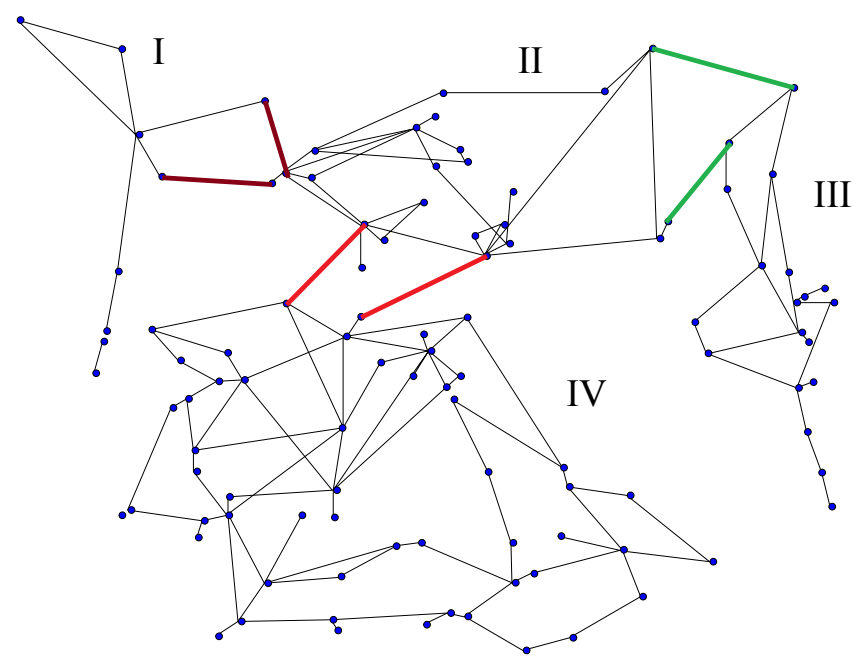

Figure 7: Detecting the most vulnerable lines and dividing the power grid into four subnets (I, II, III, and IV).

functioning of the network, for identifying a hierarchy of connections within a complex architecture, and for detecting the vulnerable links. The application of min-cut theorem over spectral analysis provides a way to find subnets. However, this method does not work properly in finding subnets in power transmission grids [5,13]. Instead, we performed a subjective assessment on the graph and found that, as shown in Figure 7, by removing only 6 out of 142 links, it becomes possible to break the $400 \mathrm{kv}$ high voltage power network into four sub nets: eastern network (I), northern network (II), western network (III), and finally southern network (IV).

There are several static and dynamic aspects that could be taken into account in the removing critical links from a network, in this paper we consider geographical distance and situation of buses for removing the links.

In this section, we attempt to analyze each of these subnets separately. In Table 5, we report the topological properties of our new born subnets.

Subnet II and III have small C and small $<\mathrm{l}>$, that behave as random networks, and are vulnerable against random failures and intentional attacks. Subnet IV behaves like the whole network, as small world network with moderate $\mathrm{C}$ and small $<\mathrm{l}>$, that are vulnerable against intentional attacks.

It is important to note that the above 6 links are the most vulnerable transmission lines in Iran's $400 \mathrm{kv}$ power grid, i.e. to improve the reliability of total network one way is to introduce more redundancy into these lines. At the same time, these links are important for reducing the chance of cascading failures, when appropriate control mechanisms are installed at automation and control layer [18].

Research in vulnerability analysis can be further advanced by adopting recent developments including the approach adopted by Chen et al. $[27,28]$ who depict a typical power grid as a weighted graph [27], and proposed a hybrid approach [28], and by Wang et al. [23], who used simulation results of the IEEE-118 bus system and the central China power grid to show that the nodes with high electrical betweenness play critical roles in both topological structure and vulnerability of power transmission grids [23]. 
Citation: M.A.S. Monfared, Alipour Z (2013) Structural Properties and vulnerability of Iranian 400kv Power Transmission Grid: a Complex Systems Approach. Ind Eng Manage 2: 112. doi:10.4172/2169-0316.1000112

\begin{tabular}{|c|c|c|c|c|c|c|c|}
\hline Subnet & $\mathbf{N}$ & $\mathbf{L}$ & $<\mathbf{>}$ & $<\mathbf{>}$ & $\mathbf{C}$ & $\mathbf{k}_{\max }$ & $\mathbf{d}$ \\
\hline $\mathbf{I}$ & 9 & 9 & 2 & 2.54 & 0.2333 & 5 & 5 \\
\hline II & 23 & 31 & 2.695 & 2.928 & 0.052 & 6 & 6 \\
\hline III & 19 & 22 & 2.316 & 3.367 & 0.00 & 4 & 8 \\
\hline IV & 54 & 74 & 2.777 & 4.699 & 0.0766 & 7 & 11 \\
\hline Total & 105 & 142 & 2.7048 & 6.8817 & 0.1097 & 7 & 19 \\
\hline
\end{tabular}

Table 5: Topology Specifications of Subnets (I, li, lii, And IV).

\section{Conclusion}

The main outcome of this research is to reemphasize the results of previous studies [2,4-6] in revealing that, despite the inherent differences, every real power transmission networks analyzed so far are characterized by the same topological properties, i.e. they display exponential degree distribution rather than power law distribution. It is evident from our results that Iran's power transmission network (at 400kvlevel), with exponential degree distribution, displays small world effects, i.e. relatively moderate clustering coefficient $(0.1097)$ much more than that for random network $(0.0257)(\mathrm{C}=\langle\mathrm{k}>/ \mathrm{N})$, and moderate value of path length (6.8817) $(\ln \mathrm{N} / \mathrm{ln}<\mathrm{k}>)$, larger than that pertaining to random networks (4.6775).We also found that Iran power transmission network like other complex networks would be sensitive to a very limited number of links (i.e. 6 links or transmission lines out of 142).

\section{Acknowledgement}

Authors wish to thank Dr. Fotouhi Firuzabadi from school of electrical engineering in Sharif University for his helps to access the data required for the analysis performed in this paper.

\section{References}

1. Amaral LAN, Ottino JM (2004) Complex systems and networks: challenges and opportunities for chemical and biological engineers. Chem Eng Sci 59: 1653-1666.

2. Rosas-Casals M, Valverde S, Sole RV (2007) Topological Vulnerabilityofthe European Power Gridunder Errorsand Attacks. Int J Bifur Chaos 17: 24652475.

3. Newman DE, Carreras BA, Degala NS, Dobson I (2012) Risk Metrics for Dynamic Complex Infrastructure Systems such as the Power Transmission Grid. 45th Hawaii International Conference on System Science Hawaii.

4. Chassin DP, Posse C (2005) Evaluating North American electric grid reliability using the Barabasi- Albert network model. Physica A 355: 667-677.

5. Rosato V, Bologna S, Tiriticco F (2007) Topological properties of high-voltage electrical transmission networks. Elec Pow Sys Res 77: 99-105.

6. Solé RV, Rosas-Casals M, Corominas-Murtra B, Valverde S (2008) Robustness of the European power grids under intentional attack. Phy Rev 77.
7. Watts DJ (1999) Small Worlds: The Dynamics of Networks between Order and Randomness. Princeton University Press.

8. Albert R, Barabasi AL (2002) Statistical mechanics of complex networks. 74

9. Lewis TG (2009) Network Science. John Wiley \& Sons

10. Hsu LH, Lin CK (2009) Graph theory and interconnection networks. CRC Press.

11. Zio E (2009) Reliability engineering: Old problems and new challenges. Relia Eng Sys Safe 94 125-141.

12. Kastenberg WE (2005) Assessing and Managing the Security of Complex Systems: Shifting the RAMS Paradigm. Proceedings of the 29th ESReDA Seminar on Systems Analysis for a more Secure World JRC-IPSC 111-126.

13. Boccaletti S, Latora V, Moreno Y, Chavez M, Hwang DU (2006) Complex networks: Structure and dynamics. Phy Rep 424: 175-308.

14. Newman MEJ (2003) The structure and function of complex networks. SIAM Rev 45: 167-256.

15. Yaneer BY (1997) Dynamics of Complex Systems. Addison-Wesley.

16. Watt DJ, Strogatz SH (1998) Collective dynamics of "small-world" networks. Nature 393: 440-442.

17. Barabasi AL, Albert R (199) Emergence of scaling in random networks. Science 286: 509-512.

18. Crucitti P, Latora V, Marchiori M (2004) A Topological Analysis of the Italian Electric Power Grid. Physica A 338: 92-97.

19. Albert R, Albert I, Nakarado GL (2004) Structural vulnerability of the North American power grid. Phys Rev E 69.

20. Sanchez JAA (2006) Dynamics and topology in complex networks. in Departamento de ciencias la naturaleza y Fisica A plicada.

21. http://www2.tavanir.org.ir/info/stat88/sanatfhtml/

22. Tian X, Chen J, He Y, He D (2004) Complex network properties of Chinese power grid. Int J Modern Phys B 18: 2599-2603.

23. Wang K, Zhang B, Zhang Z, Yin X, Wang B (2011) An electrical betweenness approach for vulnerability assessment of power grids considering the capacity of generators and load. Physica A 390: 4692-4701.

24. Surdutovich GC, Cortez, Vitilina R (2002) Dynamics of "small world" networks and vulnerability of the electric power grid. Proceedings of 8thSymposium of Specialists in Electric Operational and Expansion Planning Brazil.

25. http://www.igmc.ir/

26. http://vlado.fmf.uni-lj.si/pub/networks/pajek/

27. Chen G, Donng ZY,Hill DJ, Zhang JH (2009) An improved model for structural vulnerability analysis of power networks. Physica A 388: 4259-4266.

28. Chen, G, Dong ZY, Hill DJ zhang GH, Hua KQ (2010) Attack structural vulnerability of power grids: A hybrid approach based on complex networks. Physica A 389: 595-603. 